\title{
The Almost Intersection Property for Pairs of Matroids on Common Groundset
}

\author{
Nathan Bowler \\ University of Hamburg \\ Hamburg, Germany
}

\author{
Johannes Carmesin \\ University of Birmingham \\ Birmingham, U.K.
}

\author{
Jerzy Wojciechowski \\ West Virginia University \\ Morgantown WV, U.S.A.
}

\author{
Shadisadat Ghaderi \\ Guttman Community College \\ City University of New York \\ New York, U.S.A. \\ shadisadat .ghaderi@guttman . cuny . edu
}

Submitted: Jul 16, 2019; Accepted: Jun 1, 2020; Published: Jul 10, 2020

(C) The authors. Released under the CC BY-ND license (International 4.0).

\begin{abstract}
We introduce the Almost Intersection Property for pairs of possibly infinite matroids on the same groundset. We prove that if such a pair satisfies the Almost Intersection Property then it satisfies the Matroid Intersection Conjecture of NashWilliams. We also present some corollaries of that result.
\end{abstract}

Mathematics Subject Classifications: 05B35

\section{Introduction}

The finitary case of the following Matroid Intersection Conjecture has been introduced by Nash-Williams (see [1]). It is a generalization of the well known Edmonds' Intersection Theorem.

Conjecture 1.1. If $M$ and $N$ are matroids on a common groundset $E$, then there exist disjoint $I, J \subseteq E$ such that $\operatorname{cl}_{M}(I) \cup \operatorname{cl}_{N}(J)=E$ and $I \cup J$ is independent in both $M$ and $N$.

For the rest of this section assume that $M$ and $N$ are matroids on a common ground set $E$. A packing for $(M, N)$ is a pair $(S, T)$ of disjoint subsets of $E$ such that $\operatorname{cl}_{M}(S)=$ $\operatorname{cl}_{N}(T)=E$. A covering for $(M, N)$ is a pair $(A, B)$ of subsets of $E$ that are independent in $M, N$, respectively, and $A \cup B=E$. We say that $(M, N)$ has the Packing/Covering 
Property iff there exists a partition $E=P \sqcup C$ such that $\left(M \uparrow_{P}, N \uparrow_{P}\right)$ has a packing and $(M . C, N . C)$ has a covering. Bowler and Carmesin [3] proved the following result showing that the Matroid Intersection Conjecture is equivalent to a conjecture involving the Packing/Covering Property.

Fact 1.2 (Bowler and Carmesin [3]). (M,N) satisfies the Matroid Intersection Conjecture if and only if $\left(M, N^{*}\right)$ has the Packing/Covering Property.

We say that the pair $(M, N)$ has the Almost Intersection Property when there exist almost disjoint $I, J \subseteq E$ such that $\operatorname{cl}_{M}(I) \cup \mathrm{cl}_{N}(J)$ is almost equal to $E$ and $I \cup J$ is almost independent in both $M$ and $N$. We mean here that the sets $I \cap J, E \backslash\left(\operatorname{cl}_{M}(I) \cup \operatorname{cl}_{N}(J)\right)$ and $(I \cup J) \backslash K$ are all finite for some $K \subseteq E$ that is independent in both $M$ and $N$.

We say that $(S, T)$ is an almost packing of $(M, N)$ iff $S$ and $T$ are spanning in $M$ and $N$, respectively, and $S \cap T$ is finite. Analogously, we say that $(I, J)$ is an almost covering of $(M, N)$ iff $I$ and $J$ are independent in $M$ and $N$, respectively and $E \backslash(I \cup J)$ is finite. If there exists a partition $E=P \sqcup Q$ of $E$ such that $(M \backslash Q, N \backslash Q)$ has an almost packing and $(M / P, N / P)$ has an almost covering, then we say that $(M, N)$ has the Almost Packing/Covering Property.

If $F \subseteq E$, then say that $(M, N)$ has the Packing/Covering Property modulo $F$ iff there exists a partition $E \backslash F=P \sqcup C$ such that $(M, N) / F \backslash C$ has a packing and $(M, N) \backslash F / P$ has a covering. The following proposition will be proved in the next section.

Proposition 1.3. $(M, N)$ has the Almost Intersection Property if and only if $\left(M, N^{*}\right)$ has the Packing/Covering Property modulo a finite set.

The main result of this paper is the following theorem.

Theorem 1.4. If $(M, N)$ has the Almost Intersection Property, then it satisfies the Matroid Intersection Conjecture.

Note that Theorem 1.4 immediately implies Edmonds' Intersection Theorem (the finite case of the Matroid Intersection Conjecture).

Theorem 1.4 follows from Fact 1.2, Proposition 1.3 and the following result.

Theorem 1.5. The following are equivalent.

1. $(M, N)$ has the Packing/Covering Property.

2. $(M, N)$ has the Almost Packing/Covering Property.

3. $(M, N)$ has the Packing/Covering Property modulo a finite subset of $E$

This result also has the following corollary:

Corollary 1.6. If $(M, N)$ has the Packing/Covering Property and $A, B \subseteq E$ are finite, then $(M, N) / A \backslash B$ also has the Packing/Covering Property. 


\section{Preliminaries}

We follow the notation and terminology of [6] and [5]. In particular, the following statements about a set $\mathscr{I} \subseteq \mathscr{P}(\mathrm{E})$ are independence axioms:

(I1) $\varnothing \in \mathscr{I}$.

(I2) $\mathscr{I}$ is closed under taking subsets.

(I3) If $I, J \in \mathscr{I}$ with $I$ maximal in $\mathscr{I}$ and $J$ not maximal, there exist an $x \in I \backslash J$ such that $J \cup\{x\} \in \mathscr{I}$.

(IM) If $I \subseteq X \subseteq E$ and $I \in \mathscr{I}$, then the set $\{J \in \mathscr{I}: I \subseteq J \subseteq X\}$ has a maximal element.

When a set $\mathscr{I} \subseteq \mathscr{P}(\mathrm{E})$ satisfies the independence axioms, we call the pair $M=(E, \mathscr{I})$ a matroid on $E=E(M)$ and refer to the elements of $\mathscr{I}=\mathscr{I}(M)$ as the independent sets of $M$. The following background of matroids will be used in this paper: For $F, S \subseteq E$, we have

$S$ is spanning in $M / F$ if and only if $S \cup F$ is spanning in $M$.

Proof of Proposition 1.3. Assume first that there exists a partition $E=P \sqcup Q \sqcup F$ such that $F$ is finite, $\left(M, N^{*}\right) / F \backslash Q$ has a packing and $\left(M, N^{*}\right) \backslash F / P$ has a covering. Then $P$ and $Q$ can be partitioned as $P=S \sqcup T$ and $Q=B \sqcup A$ with $T \subseteq \operatorname{cl}_{M}(S \cup F)$, $S \subseteq \mathrm{cl}_{N^{*}}(T \cup F), A \subseteq \mathrm{cl}_{N}(B \cup F)$ and $B \subseteq \operatorname{cl}_{M^{*}}(A \cup F)$. Moreover, we can assume without loss of generality that $S$ is independent in $M$ and $B$ is independent in $N$ (see Figure 2.1).

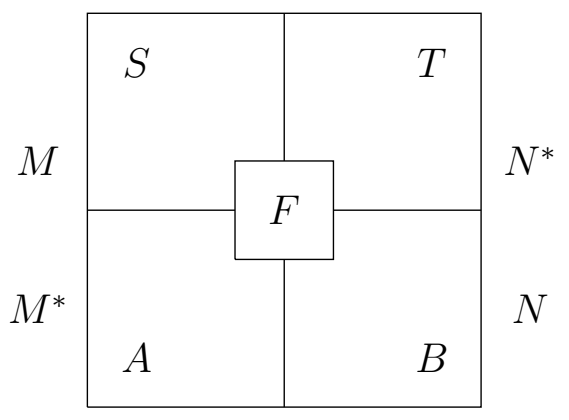

Figure 2.1: The sets $F, S, T, A$ and $B$.

Since $T \cup F$ is spanning in $N^{*} \backslash Q$, it follows that $S$ is independent in $N / Q$ implying that $S \cup B$ is independent in $N$. Similarly $S \cup B$ is independent in $M$. Let $I=S \cup F$ and $J=B \cup F$. Then $I, J$ are almost disjoint and $I \cup J$ is almost independent in both $M$ and $N$. Moreover, $\operatorname{cl}_{M}(I) \cup \operatorname{cl}_{N}(J)=E$. It follows that $(M, N)$ has the Almost Intersection Property. 
Now assume that $(M, N)$ has the Almost Intersection Property. Let $I, J \subseteq E$ be almost disjoint and such that $\operatorname{cl}_{M}(I) \cup \mathrm{cl}_{N}(J)$ is almost equal to $E$ and $I \cup J$ is almost independent in both $M$ and $N$. Without loss of generality, we can assume that $I$ is independent in $M$ and $J$ is independent in $N$. Let $I^{\prime} \subseteq I \backslash J$ and $J^{\prime} \subseteq J \backslash I$ be such that $I \cup J^{\prime}$ is a basis of $M \uparrow_{I \cup J}$ and $J \cup I^{\prime}$ is a basis of $N \uparrow_{I \cup J}$. Note that $(I \cup J) \backslash\left(I^{\prime} \cup J^{\prime}\right)$ is finite. Let $P^{\prime}, Q^{\prime} \subseteq E \backslash(I \cup J)$ be disjoint and such that $E \backslash\left(I \cup J \cup P^{\prime} \cup Q^{\prime}\right)$ is finite with $P^{\prime} \subseteq \operatorname{cl}_{M}(I)$ and $Q^{\prime} \subseteq \operatorname{cl}_{N}(J)$. Let $P=I^{\prime} \cup P^{\prime}, Q=J^{\prime} \cup Q^{\prime}$ and $F=E \backslash(P \cup Q)$ (see Figure 2.2).

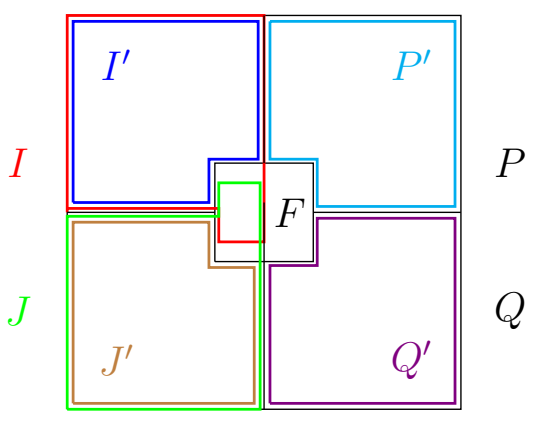

Figure 2.2: A packing for $\left(M, N^{*}\right) / F \backslash Q$ and a covering for $\left(M, N^{*}\right) \backslash F / P$.

Note that $F$ is finite and $P \subseteq \operatorname{cl}_{M}\left(I^{\prime} \cup F\right)$. Moreover, since $I^{\prime} \cup J$ is independent in $N$ and $J$ spans every element of $Q$, it follows that $I^{\prime}$ is independent in $N / Q$ and hence $P^{\prime} \cup F$ is spanning in $(N / Q)^{*}=N^{*} \backslash Q$. It follows that $\left(I^{\prime}, P^{\prime}\right)$ is a packing for $\left(M, N^{*}\right) / F \backslash Q$. Similarly, $\left(J^{\prime}, Q^{\prime}\right)$ is a packing for $\left(N, M^{*}\right) / F \backslash P$ and hence it is a covering for $\left(M, N^{*}\right) \backslash F / P$.

\section{The Main Result}

Throughout this section we assume that $M$ and $N$ are matroids on a common ground set E.

A semi-packing for $(M, N)$ is a pair $(B, D)$ of subsets of $E$ that are spanning in $M$ and $N$, respectively, with a minimal possible intersection. More precisely, if $\left(B^{\prime}, D^{\prime}\right)$ is another pair of subsets of $E$ that are spanning in $M$ and $N$, respectively, and $B^{\prime} \cap D^{\prime} \subseteq B \cap D$ then $B^{\prime} \cap D^{\prime}=B \cap D$. A semi-covering for $(M, N)$ is defined analogously as a pair of independent subsets with a maximal possible union. It is easy to see the following result:

Lemma 3.1. Let $F \subseteq E$ be finite. We have the followings:

1. If $(M, N)$ has an almost packing, then $(M, N)$ has a semi-packing.

2. If $(M, N)$ has an almost covering, then $(M, N)$ has a semi-covering.

3. If $(M, N) / F$ has a packing, then $(M, N)$ has an almost packing. 


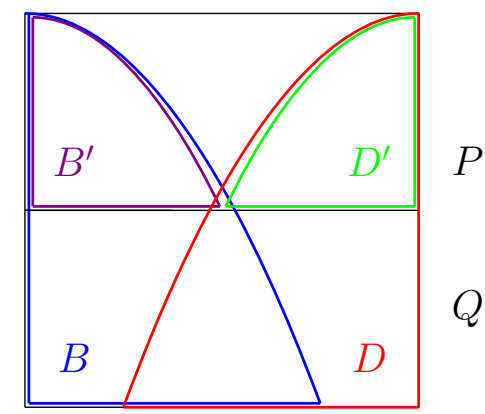

Figure 3.1: The sets $B, D, B^{\prime}$ and $D^{\prime}$.

4. If $(M, N) \backslash F$ has a covering, then $(M, N)$ has an almost covering.

Let $B$ and $D$ be independent in $M$ and $N$, respectively. A $(B, D)$-exchange $M$-chain is a finite sequence $\left(e_{1}, e_{2}, \ldots, e_{n}\right)$ of elements of $E$ such that for each $i \in\{1,2, \ldots, n-1\}$ the elements $e_{i}$ and $e_{i+1}$ are distinct and:

- if $i$ is odd, then there exists a circuit $C$ of $M$ with $e_{i}, e_{i+1} \in C \subseteq B \cup\left\{e_{i}\right\}$;

- if $i$ is even, then there exists a circuit $C$ of $N$ with $e_{i}, e_{i+1} \in C \subseteq D \cup\left\{e_{i}\right\}$.

We say that such a chain is from $e_{1}$ to $e_{n}$.

A $(B, D)$-exchange $N$-chain is defined analogously with the words "even" and "odd" interchanged. A $(B, D)$-exchange chain refers to either of these notions.

The following lemma is proved in [2].

Lemma 3.2. Let $B$ and $D$ be independent in $M$ and $N$, respectively. If there exists a $(B, D)$-exchange $M$-chain from $d \in E \backslash(B \cup D)$ to $e \in B \cap D$, then there exist $B^{\prime}$ and $D^{\prime}$ that are independent in $M$ and $N$, respectively, such that $B^{\prime} \cap D^{\prime}=(B \cap D) \backslash\{e\}$, $\mathrm{cl}_{M}(B) \subseteq \mathrm{cl}_{M}\left(B^{\prime}\right)$ and $\mathrm{cl}_{N}(D) \subseteq \mathrm{cl}_{N}\left(D^{\prime}\right)$.

The following lemma is the key technical result that will be used in the proof of the main result.

Lemma 3.3. If $(M, N)$ has a semi-packing, then it has the Packing/Covering Property.

Proof. Let $(B, D)$ be a semi-packing for $(M, N)$. Without loss of generality, we can assume that $B$ and $D$ are bases of $M$ and $N$, respectively. Let $B^{\prime}$ be the set of all $e \in B$ to which there exists a $(B, D)$-exchange chain from an element of $E \backslash(B \cup D)$. Similarly, let $D^{\prime}$ consist of those $e \in D$ to which there exists a $(B, D)$-exchange chain from $E \backslash(B \cup D)$ (see Figure 3.1).

Note that $B^{\prime} \cap D=\varnothing$ and $D^{\prime} \cap B=\varnothing$, since if $e \in B \cap D$ and there is a $(B, D)$ exchange $M$-chain from $d \in E \backslash(B \cup D)$ to $e$, then Lemma 3.2 implies that there exist $B^{\prime \prime}$ and $D^{\prime \prime}$ that are bases of $M$ and $N$, respectively, such that

$$
B^{\prime \prime} \cap D^{\prime \prime}=(B \cap D) \backslash\{e\} .
$$


Since $B^{\prime \prime} \cap D^{\prime \prime}$ is a proper subset of $B \cap D$ and $(B, D)$ is a semi-packing we get a contradiction. Similarly, the existence of a $(B, D)$-exchange $N$-chain would lead to a contradiction.

Let

$$
P=B^{\prime} \cup D^{\prime} \cup(E \backslash B \cup D),
$$

(see Figure 3.1). We claim that $\left(B^{\prime}, D^{\prime}\right)$ is a packing for $\left(M \uparrow_{P}, N \uparrow_{P}\right)$. If $e \in E \backslash(B \cup D)$, then the definition of exchange chains implies that $e$ is spanned by $B^{\prime}$ in $M$ and by $D^{\prime}$ in $N$. If $e \in B^{\prime}$, then $e$ is spanned by $D$ in $N$ so there exists a circuit $C$ of $N$ with $\{e\} \in C \subseteq D \cup\{e\}$. Since $e \in B^{\prime}$, it follows that there exists a $(B, D)$-exchange chain from $E \backslash(B \cup D)$ to each element of $C \backslash\{e\}$ implying that $C \backslash\{e\} \subseteq D^{\prime}$. Thus $e$ is spanned by $D^{\prime}$ in $N$. Similarly, each element of $D^{\prime}$ is spanned by $B^{\prime}$ in $M$ completing the proof that $\left(B^{\prime}, D^{\prime}\right)$ is a packing for $\left(M \uparrow_{P}, N \uparrow_{P}\right)$.

Let $Q=E \backslash P$ (see Figure 3.1). $\hat{B}=B \cap Q$ and $\hat{D}=D \cap Q$. We claim that $(\hat{B}, \hat{D})$ is a covering of $(M . Q, N . Q)$. Clearly $\hat{B} \cup \hat{D}=Q$. Since $B=B^{\prime} \cup \hat{B}$ is independent in $M$ and $B^{\prime}$ spans $P$ in $M$, it follows that $\hat{B}$ is independent in M.Q. Similarly, $\hat{D}$ is independent in N.Q completing the proof of the lemma.

Since $(M, N)$ has the Packing/Covering Property if and only if $\left(M^{*}, N^{*}\right)$ does, we also get the following corollary:

Corollary 3.4. If $(M, N)$ has a semi-covering, then it has the Packing/Covering Property.

The proof of the following lemma is routine.

Lemma 3.5. Let $I$ be independent in $M$ and $F \subseteq E$ be finite. Then there exists $I^{\prime} \subseteq I$ that is independent in $M / F$ with $I \backslash I^{\prime}$ finite.

Corollary 3.6. If $(M, N)$ has an almost covering and $F \subseteq E$ is finite, then $(M / F, N / F)$ has an almost covering.

Now we are ready to prove Theorem 1.5.

Proof of Theorem 1.5. It is clear that 1. implies 2., which implies 3. It suffices to show that 3. implies 1 .

Assume that $(M, N)$ has the Packing/Covering Property modulo finite $F \subseteq E$. Let $P \sqcup Q$ be a partition of $E \backslash F$ such that $(M, N) / F \backslash Q$ has a packing and $(M, N) \backslash F / P$ has a covering. Since $F$ is finite, it follows that $(M \backslash Q, N \backslash Q)$ has an almost packing and hence it has a semi-packing. Consequently, Lemma 3.3 implies that $(M \backslash Q, N \backslash Q)$ has the Packing/Covering Property. Let $E \backslash Q=P^{\prime} \sqcup Q^{\prime}$ be a partition of $E \backslash Q$ such that $\left(M \uparrow_{P^{\prime}}, N \uparrow_{P^{\prime}}\right)$ has a packing $(S, T)$ and $(M, N) \backslash Q / P^{\prime}$ has a covering $(A, B)$ (see Figure 3.2). 


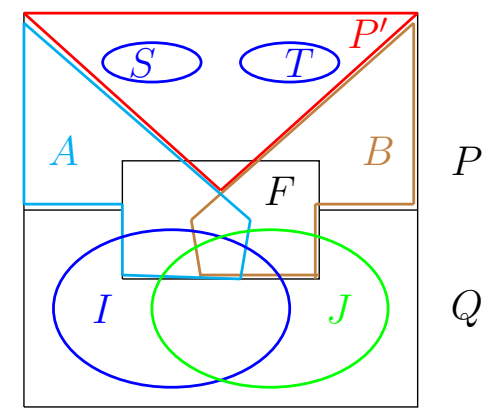

Figure 3.2: A packing $(S, T)$ for $\left(M \uparrow_{P^{\prime}}, N \uparrow_{P^{\prime}}\right)$ and a covering $(A, B)$ for $(M, N) \backslash Q / P^{\prime}$.

A covering of $(M, N) \backslash F / P$ is an almost covering of $(M / P, N / P)$ and $P^{\prime} \backslash P$ is finite so Corollary 3.6 implies that $\left(M /\left(P \cup P^{\prime}\right), N /\left(P \cup P^{\prime}\right)\right)$ has an almost covering $(I, J)$. Since $F$ is finite, it follows that $((A \cup I) \backslash F,(B \cup J) \backslash F)$ is an almost covering of $\left(M / P^{\prime}, N / P^{\prime}\right)$. Since $\left(M / P^{\prime}, N / P^{\prime}\right)$ has a semi-covering, Corollary 3.4 implies that it has the Packing/Covering Property.

Let $P^{\prime \prime} \sqcup Q^{\prime \prime}$ be a partition of $E \backslash P^{\prime}$ such that $(M, N) / P^{\prime} \backslash Q^{\prime \prime}$ has a packing $\left(S^{\prime}, T^{\prime}\right)$ and $(M, N) / P^{\prime} / P^{\prime \prime}$ has a covering $\left(A^{\prime}, B^{\prime}\right)$ (see Figure 3.3).

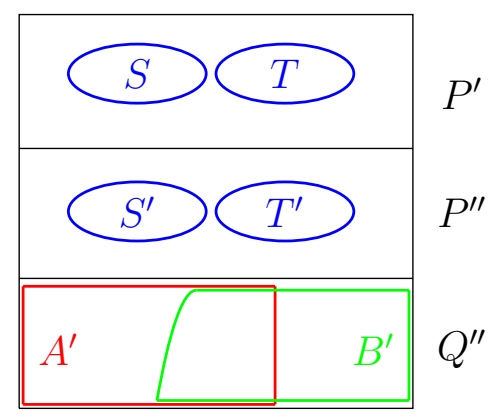

Figure 3.3: A packing $\left(S^{\prime}, T^{\prime}\right)$ for $(M, N) / P^{\prime} \backslash Q^{\prime \prime}$ and a covering $\left(A^{\prime}, B^{\prime}\right)$ for $(M, N) / P^{\prime} / P^{\prime \prime}$.

Then $\left(S \cup S^{\prime}, T \cup T^{\prime}\right)$ is a packing for $\left(M \backslash Q^{\prime \prime}, N \backslash Q^{\prime \prime}\right)$ implying that $(M, N)$ has the Packing/Covering Property.

\section{Consequences}

Our results imply the following strengthening of Edmonds' Intersection Theorem.

Corollary 4.1. If $M$ has finite rank and $N$ is arbitrary, then $(M, N)$ satisfies the Matroid Intersection Conjecture. 
Proof. Let $M$ be a matroid of finite rank, and $N$ be an arbitrary matroid. Observe that $\left(M^{*}, N\right)$ has an almost covering. Hence $\left(M^{*}, N\right)$ has a semi-covering and Corollary 3.4 implies that $\left(M^{*}, N\right)$ has the Packing/Covering Property. By Fact 1.2 the pair $(M, N)$ satisfies the Matroid Intersection Conjecture.

A matroid $M$ on $E$ is nearly finitary iff for every $A \subseteq E$ that contains no finite circuits of $M$ there exists a finite $F \subseteq A$ such that $A \backslash F$ is independent in $M$. Assume that $M$ and $N$ are matroids on the same ground set $E$. Let $M \vee N$ be the set system $M \vee N=(E, \mathscr{I}(M \vee N))$, where

$$
\mathscr{I}(M \vee N)=\{I \cup J: I \in \mathscr{I}(M), J \in \mathscr{I}(N)\} .
$$

The following result is proved in [2].

Theorem 4.2. If $M$ and $N$ are nearly finitary, then $M \vee N$ is a nearly finitary matroid.

In [2] it is proved that if $M \vee N^{*}$ is a matroid, then $(M, N)$ satisfies the Intersection Conjecture. In particular, the following result holds. We can use Corollary 3.4 to provide an alternative proof.

Theorem 4.3. If $M$ and $N$ are nearly finitary, then $(M, N)$ has the Packing/Covering Property.

Proof. By Theorem 4.2, $M \vee N$ is a matroid. If $I$ and $J$ are independent in $M$ and $N$, respectively, with $I \cup J$ being a basis of $M \vee N$, then $(I, J)$ is a semi-covering of $(M, N)$. By Corollary 3.4, $(M, N)$ has the Packing/Covering Property.

In [4] "patchwork matroids" are introduced and proved to satisfy the following characterization. Here $K \triangle B=(K \backslash B) \cup(B \backslash K)$.

Theorem 4.4. The matroid $M$ is patchwork if and only if for every $K \subseteq E$ one of the following conditions holds:

1. $K$ is independent in $M$.

2. $K$ is spanning in $M$.

3. There exists a basis $B$ of $M$ with finite $K \triangle B$.

Lemma 3.3 implies the following result.

Theorem 4.5. If $M$ is patchwork and $N$ is arbitrary, then $(M, N)$ has the Packing/ Covering Property.

Proof. Let $D$ be a basis of $N$ and $K=E \backslash D$. If $K$ is independent in $M$, then $(M, N)$ has a covering. If $K$ is spanning in $M$, then $(M, N)$ has a packing. Otherwise, by Theorem 4.4, there exists a basis $B$ of $M$ with finite $K \triangle B$. Then $(B, D)$ is an almost packing for $(M, N)$. Hence $(M, N)$ has a semi-packing and Lemma 3.3 implies that $(M, N)$ has the Packing/Covering Property. 


\section{References}

[1] R. Aharoni and R. Ziv, The intersection of two infinite matroids, Journal of the London Mathematical Society 58 (1998) 513-525.

[2] E. Aigner-Horev, J. Carmesin, and J. Fröhlich, On the intersection of infinite matroids, Discrete Mathematics 341 (2018) 1582-1596.

[3] N. Bowler and J. Carmesin, Matroid intersection, base packing and base covering for infinite matroids, Combinatorica 35 (2015), 153-180.

[4] N. Bowler and M. Storm, Patchwork matroids, preprint.

[5] H. Bruhn, R. Diestel, M. Kriesell, R. Pendavingh and P. Wollan, Axioms for infinite matroids, Advances in Mathematics 239 (2013), 18-46.

[6] J. Oxley, Matroid Theory, Oxford University Press, Inc. 2011. 